\title{
Challenge of COVID 19: Pathogenicity, Genetic Variations and Laboratory Diagnosis
}

\author{
Eltayib H Ahmed-Abakur ${ }^{1,2 *}$ \\ ${ }^{1}$ Department of Microbiology and Immunology, Zaiem AlAzhari University, Sudan \\ ${ }^{2}$ Department of Medical Laboratory Sciences, University of Tabuk, Saudi Arabia
}

*Corresponding author: Eltayib H Ahmed-Abakur, Department of Microbiology and Immunology, Alzaiem AlAzhari University, Faculty of Medical Laboratory Sciences, Khartoum North, Sudan.

Department of Medical Laboratory Sciences, Faculty of Applied Medial Sciences, University of Tabuk, Tabuk, Saudi Arabia.

To Cite This Article: Eltayib H Ahmed-Abakur. Challenge of COVID 19: Pathogenicity, Genetic Variations and Laboratory Diagnosis. 2020 11(1). AJBSR.MS.ID.001604. DOI: 10.34297/AJBSR.2020.11.001604.

Received: 眥 November 24, 2020; Published: 觜 December 08, 2020

\begin{abstract}
At the time of preparing this report Coronavirus 2019 disease (COVID 19) spreads to 220 countries and territories around the world, infected 60 million person with 1405197 reported deaths. This review focused on challenge concerned pathogenicity (transmission and clinical pictures), genetic variations and laboratory diagnosis of COVID 19. Systematic rapid review was conducted by searched the PubMed and Google Scholar with search terms including SARS-COV-2, COVID-19, genetic variations, laboratory diagnosis and pathogenicity. The retrieved data showed huge genetic variations, broad ways of transmission, non-specific symptoms of COVID 19 and difficulty in conclusion.

Conclusion: Awareness of genetic variations, possible transmission ways and weakness of the current laboratory diagnostic tools of COVID 2019 may contribute to understand variability of COVID-19 and help in improve diagnostic tools.
\end{abstract}

Keywords: COVID19; Pathogenicity; Genetic variations; Laboratory diagnosis

\section{Introduction}

The World Health Organization declared the coronavirus disease 2019 (COVID-19) as pandemic disease due to it's the widespread infectivity and high contagion rate [1]. During the past two decades three coronaviruses (CoVs) emerged as a global health emergency epidemic diseases (COVID-19 in 2019, Middle East respiratory syndrome (MERS) in 2012, and severe acute respiratory syndrome (SARS) in 2002) [1-4]. Coronaviruses (CoVs) also recognized in various hosts of mammalians such as Camelus sp., Paguma larvata, Felis catus, bats and birds [3]. This scenario of multi-epidemic CoVs diseases in short periods and diversity of virus hosts which may encourages genetic modification, suggesting more powerful waves of CoVs infections.

\section{Pathogenicity (Transmission and Clinical Pictures)}

A major role in the pathogenicity of COVID 19 is owing to angiotensin converting enzyme (ACE2) expression level, as it is entry receptor of the virus [5-9]. The ACE2 receptors noticeably expressed in epithelial cells of the nasal cavity, type two alveolar cells of lungs, heart, liver, kidney, gastrointestinal tract, testis and brain. The disease primary affects respiratory tract where lungs are more susceptible to COVID19 [6,7], infection may spread to other organs depends on viral load and regulation by the ACE2 receptor $[6,10]$.

The transmission of SARS-CoV-2 occurs through secretions, droplets, faecal-oral contamination $[8,11,12]$ or consumption of infected meat $[7,8]$, there is no evidence of vertical transmission $[8,13]$ although a diagnosis of COVID-19 was made in a newborn few hours after delivery from infected pregnant woman suggesting that its possibility cannot be completely excluded [13]. Moreover, the transmission of the SARS-CoV-2 virus is expected from asymptomatic, pre-symptomatic and symptomatic patients [6]. The change of seasons affect the transmission of virus [14], however several reports suggested that SARS-CoV-2 outbreak will reduced in summer season because of temperature rising, unfortunately, recent 
reports confirmed continuous transmission of SARS-CoV infections in summer [3]. In the light of current situation of highly contagion disease which is not affecting by environment conditions and broad ways of transmission particularly asymptomatic which accounts for up to $80 \%$ of the transmission [4], SARS-CoV-2 is critically threating the live-hood and may turn in to global endemic disease unless effective vaccine and specific treatment are developed.

The general symptoms of COVID-19 are cough, fever and shortness of breath $[8,14,15]$. Other symptoms include headaches, nasal congestion, sore throat, myalgia, body aches, nausea, vomiting, diarrhoea and abdominal pain $[14,15]$. Wong et al. [15] stated that smell and taste dysfunction may be the only symptom and should be added to the list of symptoms when screening for COVID19. Similarly, Agyeman et al. [16] 2020 reported that routine screening for smell and taste dysfunction conditions could contribute to improved case detection in the ongoing COVID-19pandemic. However, the symptoms of COVID-19 are nonspecific.

\section{Genetic Variations}

Knowledge of genomic interindividual variability could explain the discrepancies of spread, severity, and mortality of COVID-19 [17,18]. Debnath et al. [18] proposed three genetic gateways to COVID-19 infection; variations within ACE2 gene, the human leukocyte antigen locus and genes regulating toll-like receptor, complement pathways and subsequently cytokine storm induced exaggerated inflammatory pathways. Strafella et al. [5] determined the genetic variability of ACE2 in term single nucleotide variants (SNV) and stated five SNVs (rs2285666, rs773676270, rs774469453 and rs35803318) whereas Hou et al. [19] suggested that ACE2 or TMPRSS2 DNA polymorphisms likely associated with genetic susceptibility of COVID-19. Ahmed-Abakur \& Alnour [20] determined the genetic variations among SARS-CoV-2 strains isolated in China and showed 10 different variants. However, SARSCoV-2 has evolved into two strains designated as $\mathrm{S}$ and $\mathrm{L}$ strains. $\mathrm{L}$ strain is more aggressive and was predominant during early stages of the epidemic in Wuhan [4]. The average evolutionary rate for CoVs as a typical RNA virus is roughly 10-4 nucleotide substitutions per site per year, with mutations happening during each replication cycle. The average evolutionary rate for SARSCoV-2 is roughly $8 \times 10-4$ nucleotide substitutions per site per year, with one mutation happening in two weeks [14]. This huge genetic variations may interpret the discrepancies of transmission and severity of disease, therefore further deep study are required to elucidate the exact role of genetic variations.

\section{Laboratory Diagnosis}

RT-qPCR is the most general, effective method for detecting pathogenic viruses in blood and respiratory secretion, it is known to be highly specific $[4,21]$, but its sensitivity rate is not and estimated to be around $66-80 \%$ [22]. This technique is complicated, costly, suited only to large, centralized diagnostic centers, many reports shown false-negative RT-PCR as well the results take long time $[4,21,23]$. Other a problems may arising from sample operation, patient retention and biological safety hazards [4,21]. Moreover, certain specific swabs such as those having calcium alginate, cotton or wood contain material that inhibits PCR assays and therefore are not recommended to use for the collection of viral loaded samples. 15. As the result few rich countries have up-scaled this test on massive population whereas the poor countries have prioritized testing for definite groups of patients [4]. To overcome these obstacles, point of care technologies and serologic immunoassays (IgG/IgM and immunosorbent assay [11,14,21] are rapidly developing but currently WHO does not recommend the use of antigen-detecting rapid diagnostic tests for clinical decision making [4], thus there is strong need to expand the testing facilities, search for simple, safe, low-cost and suggestive laboratory parameters to assist in confirming COVID-19 diagnosis [11,24].

To date, there is no evidence of any effective treatment or vaccine for COVID-19 [1,14]. The best approaches for managing human CoVs are to establish a steady system of public health surveillance combined with rapid reliable diagnostic testing $[8,13,24]$.

\section{Conclusion}

More attention and studies are required to understand the role of genetic variations, break the chain of transmission, and provide cheap and simple laboratory tests.

\section{References}

1. Pascarella G, Strumia A, Piliego C, Bruno F, Del Buono R, et al. (2020) COVID-19 diagnosis and management: a comprehensive review. J Intern Med 288(2): 192-206.

2. Di Maria E, Latini A, Borgiani P (2020) Genetic variants of the human host influencing the coronavirus-associated phenotypes (SARS, MERS and COVID-19): rapid systematic review and field synopsis. Hum Genomics 14(1): 30.

3. Abdelghany TM, Ganash M, Bakri MM, Elhussieny NI, Qanash $\mathrm{H}$, et al. (2020) A review SARS-CoV-2 the other face to SARS-CoV and MERS-CoV: About future predictions. Biomedical Journal.

4. Tripathi SC, Deshmukh V, Patil A, Tripath JP (2020) COVID 19 diagnostic multiplicity and its role in community surveillance and control. Infez Med 28(Suppl 1): 18-28.

5. Strafella C, Caputo V, Termine A (2020) Analysis of ACE2 genetic variability among populations highlights a possible link with COVID-19related neurological complications. Genes (Basel) 11(7): 741.

6. Loganathan S, Kuppusamy M, Wankhar W (2020) Angiotensinconverting enzyme 2 (ACE2): COVID 19 gate way to multiple organ failure syndromes. Respir Physiol Neurobiol 283: 103548.

7. Bandyopadhyay AR, Chatterjee D, Ghosh K, Sarkar P (2020) COVID 19: An epidemiological and host genetics appraisal. Asian Journal of Medical Sciences 11(5): 116-117.

8. Boregowda U, Gandhi D, Jain N, Khanna K, Gupta N (2020) Comprehensive literature review and evidence evaluation of experimental treatment in COVID 19 contagion. Clinical Medicine Insights: Circulatory, Respiratory and Pulmonary Medicine 14: 1-7. 
9. Ghafouri-Fard S, Noroozib R, Vafaeec R, Branickib W, Pośpiechb E, et al (2020) Effects of host genetic variations on response to, susceptibility and severity of respiratory infections. Biomed Pharmacother 128: 110296.

10. Mishra AK, Sahu KK, George AA, Lal A (2020) A review of cardiac manifestations and predictors of outcome in patients with COVID - 19. Heart Lung 49(6): 848-852.

11. Toledo SL, Nogueira LS, Carvalho MG, Rios DR, Pinheiro MB (2020) COVID-19: Review and hematologic impact. Clin Chim Acta 510: 170176.

12. Ovsyannikova IG, Haralambieva IH, Crooke SN, Poland GA, Kennedy RB (2020) The role of host genetics in the immune response to SARSCoV-2 and COVID-19 susceptibility and severity. IMR 202: 205-219.

13. Oda Y (2020) COVID-19: review of case reports. J Anesth 2020: 1-4.

14. Sofi MS, Hamid A, Bhat SU (2020) Coronavirus COVID- 19: A critical review of its history, pathogenesis, transmission, diagnosis and treatment. Biosafety and Health 2020: 11.002.

15. Wong DKC, Gendeh HS, Thong HK, Lum SG, Gendeh BS, et al. (2020) A review of smell and taste dysfunction in COVID-19 patients. Med J Malaysia 75(5): 574-581

16. Agyeman AA, Chin KL, Landersdorfer CB, Liew D, Ofori-Asenso R (2020) Smell and taste dysfunction in patients with COVID-19: A Systematic review and meta-analysis. Mayo Clin Proc 95(8): 1621-1631.

17. Vasku A (2020) Covid-19 infection and the host genetic predisposition: does it exist? Physiol Res 69(3): 511-514.
18. Debnath M, Banerjee M, Berk M (2020) Genetic gateways to COVID-19 infection: Implications for risk, severity, and outcomes. The FASEB Journal 34(7): 8787-8795.

19. Hou Y, Zhao J, Martin W, Kallianpur A, Chung MK, et al. (2020) New insights into genetic susceptibility of COVID-19: an ACE2 and TMPRSS2 polymorphism analysis. BMC Medicine 18: 216.

20. Ahmed-Abakur EH, Alnour TMS (2020) Genetic variations among SARS$\mathrm{CoV}-2$ strains isolated in China.

21. Huldani Uinarni H, Sukmana BI, Tommy T, Said MF, Edyson Eso A, et al. (2020) Corona Virus Infectious Disease 19 (COVID-19). Various Reviews Syst Rev Pharm 6(11): 842-857.

22. Moulahouma H, Ghorbanizamania F, Zihnioglua F, Turhanb K, Timura S (201) How should diagnostic kits development adapt quickly in COVID 19-like pandemic models? Pros and cons of sensory platforms used in COVID-19 sensing. Talanta 222: 121534.

23. Deep A, Swaroop S, Kumar A (2020) Review article: Coronavirus Disease (COVID-19) Outbreak and Adaptive Immune System of the Body. Journal of Clinical and Diagnostic Research 14(10): DE06-DE116 6.

24. Pascarella G, Strumia A, Piliego C, Buono RD, Costa F, et al. (2020) COVID-19 diagnosis and management: a comprehensive review. JIM 288(2): 192-206 